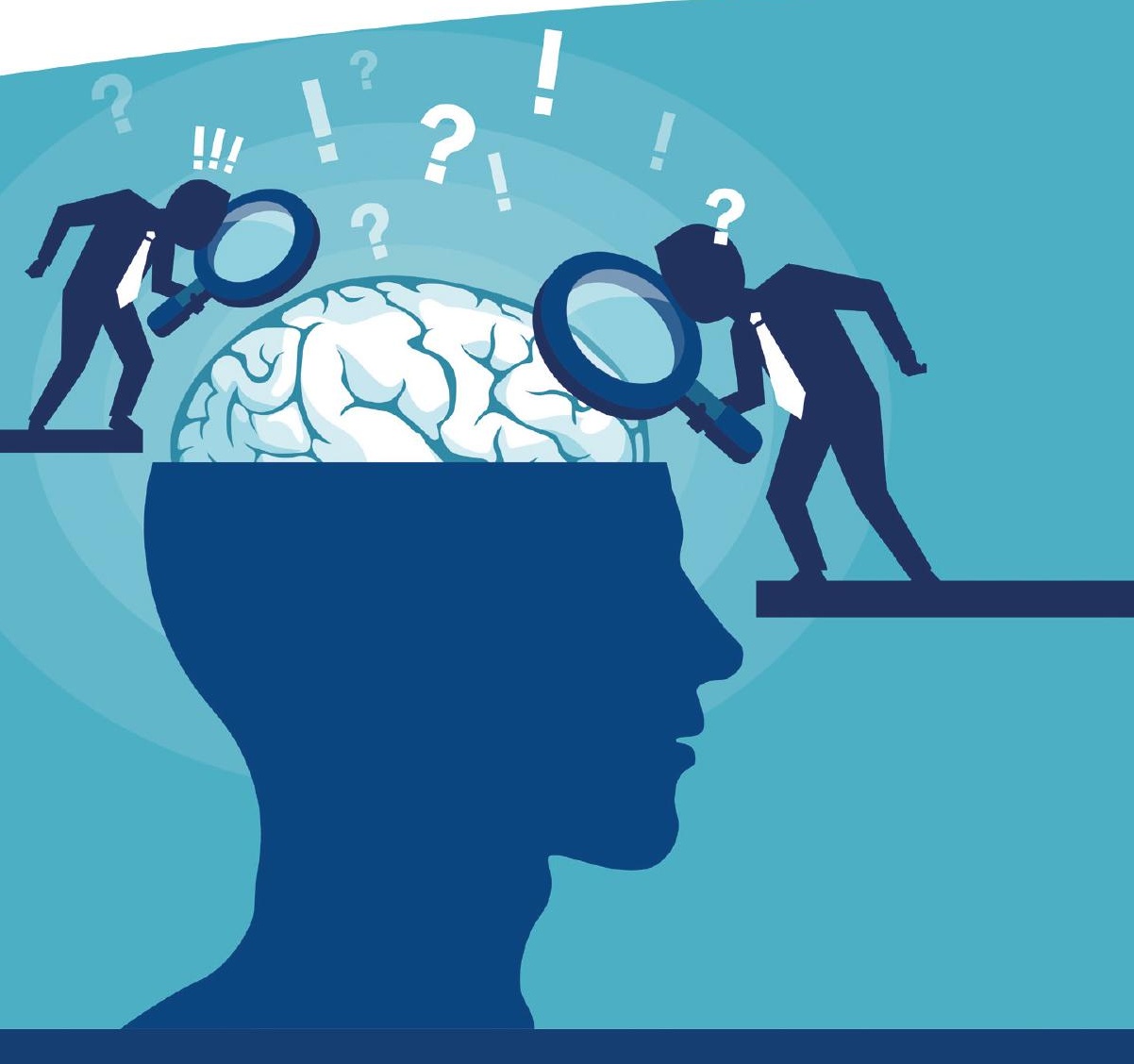

Karin Weingartz-Perschel

\title{
Mythos Genie
}

Die intellektuelle Erfahrung des Mangels 
Karin Weingartz-Perschel

Mythos Genie 
Karin Weingartz-Perschel

\section{Mythos Genie}

Die intellektuelle Erfahrung des Mangels

Tectum Verlag 
Karin Weingartz-Perschel

Mythos Genie

Die intellektuelle Erfahrung des Mangels

(C) Tectum - ein Verlag in der Nomos Verlagsgesellschaft, Baden-Baden 2019

E-Book 978-3-8288-7200-4

(Dieser Titel ist zugleich als gedrucktes Werk unter der ISBN

978-3-8288-4292-2 im Tectum Verlag erschienen.)

Umschlaggestaltung: Tectum Verlag, unter Verwendung

des Bildes \# 1188871114 von FGC | www.shutterstock.com

Alle Rechte vorbehalten

Besuchen Sie uns im Internet

www.tectum-verlag.de

Bibliografische Informationen der Deutschen

Nationalbibliothek

Die Deutsche Nationalbibliothek verzeichnet diese Publikation in der Deutschen Nationalbibliografie; detaillierte bibliografische Angaben sind im Internet über http://dnb.d-nb.de abrufbar. 


\section{Inhaltsverzeichnis}

Vorwort ......................................................................... VII

Einleitung .........................................................

I. Grundkonflikte der Intellektuellen .............................. 7

II. Die Besonderheit libidinöser Erfahrung .......................... 17

1. Bedürfnisstruktur ........................................ 17

2. Der Organismus ............................................ 20

3. Lust als Transfer ............................................ 23

III. Gibt es ein geschlechtsspezifisches Bewusstsein? .................... 27

1. Genetische Voraussetzungen ................................... 27

2. Soziale Bedingungen ......................................... 31

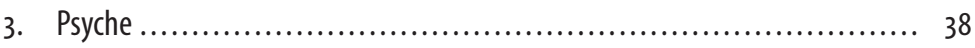

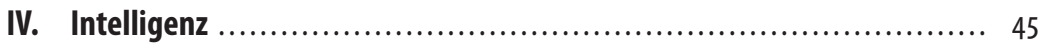

V. Das Geschlechterverhältnis ..................................... 57

1. Die Liebe.................................................. 64

2. Die Schönheit................................................... 70

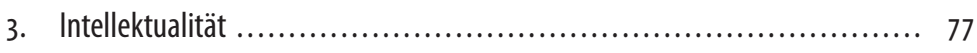

4. Der Fall Hannah Arendt.......................................... 83 
VI. Zum Verhältnis von Libido und Ratio im Spiegel der Klassen ............. 95

1. Unterschicht (Proletariat) ........................................ 97

2. Oberschicht (Bourgeoisie) ............................................ 99

3. Mittelschicht (Kleinbürgertum) ................................... 102

VII. Der sogenannte Intellektuelle .................................... 107

1. Mögliche Vielfalt der Einordnungen................................ 107

2. Ein semiotischer Versuch der Begriffsannäherung ...................... 122

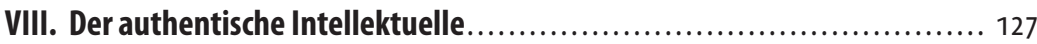

IX. Plädoyer für die Intellektuellen...................................... 141

X. Drei Beispiele typisch intellektueller Werdegänge .................... 147

1. Karl R. Popper ................................................ 148

2. Theodor W. Adorno .......................................................... 158

3. Jürgen Habermas ............................................. 172

Literaturliste.............................................................. 183 


\section{Vorwort}

Seit Langem beschäftigt mich die Frage, was wohl hinter dem Begriff "Genie“ steckt und warum so viele Menschen mit der Erklärung zufrieden sind, dass exponierte Intellektuelle, wie z. B. Adorno, Horkheimer, Habermas bis hin zu Aristoteles und Platon, einfach als Genies zu bezeichnen sind, weil sie eben von Geburt an mit besonderem kognitiven Leistungsvermögen ausgestattet sind und deshalb über ganz besondere Begabungen verfügen; dass es wie ein Schicksal zu betrachten ist, ein Genie zu sein, ebenso wie die reflexive Durchschnittlichkeit oder gar Minderbegabung der meisten anderen Menschen; dass diese Genies hochgeachtet und bewundert werden sollten, weil sie für die Gesellschaft ganz Besonderes leisten, ihr große Achtung verschaffen, sie weiterbringen - und das eben ihr Schicksal sei.

Während meines Studiums der Geschichte, Germanistik, Philosophie, Soziologie und auch der Kunst, habe ich mir in zunehmendem Maße die Frage gestellt, wie denn ein Mensch zum Intellektuellen wird. Viele Professoren habe ich währenddessen persönlich kennengelernt. Ernst Bloch, Herbert Marcuse, Leo Kofler, Wolfgang Schluchter waren u. a. darunter. Mit Habermas, Adorno, Horkheimer, Freud, Lukács, Kant, Hegel, Marx und vielen anderen habe ich mich theoretisch eindringlich auseinandergesetzt, immer auch unter der Prämisse, den Ursprung ihrer Besonderheit zu entdecken. Während der persönlichen Begegnungen kamen mir die Professoren wie ganz normale Menschen vor, die über einen enormen Kenntnisstand verfügten und diesen rhetorisch wie schriftlich überzeugend vermitteln konnten. Als ich schließlich wagte, sie nach ihrem Bildungsweg und nach ihrer Sozialisation zu fragen, wurden sie wortkarger und schnell vom Thema ablenkend, sodass ich mir nur ein grobes, etwas diffuses Bild davon machen konnte. Meistens sprachen sie dann von ihren eigenen Lehrern, denen sie ihren Werdegang verdanken. Über ihr Elternhaus und ihre Erziehung redeten sie kaum und wenn doch, dann zurückhaltend und meist mit positivem Unterton. Über ihre psycho-sozialen Probleme in 
der Zeit des Heranwachsens erfuhr ich nicht wirklich etwas. Ich wagte auch nicht, sie dahingehend zu Auskünften zu drängen. Also ging ich in ihren Schriften auf die Suche nach der Axiomatik ihrer Theorien und auch nach persönlichen Äußerungen. Außerdem verschaffte ich mir Biografien über meine Professoren und auch über diejenigen, welche ich nicht persönlich kennengelernt habe. Zu meiner Enttäuschung fand ich darin nur wenig wirklich Aufklärendes über sie. Meistens wurde versucht, ihren Werdegang als genialisch zu umschreiben. Selbst wenn Unmoralisches, Unrecht, Egozentrik, Eitelkeiten und Arroganz aufgedeckt wurden, entschuldigten die Biografen diese oft mit den Begleiterscheinungen einer genialen Persönlichkeit.

Besonders wenn es um die Phase des Heranwachsens geht, wird diese stets unter dem Tenor "Genius im Werden“ beschrieben. Unter diesem Titel trug Paul Elbogen Informationen über „Die Jugend großer Menschen “ in zweiundachtzig versuchten Porträts zusammen. ${ }^{1}$ Die meisten dieser Porträts behandeln in der Mehrzahl künstlerische sogenannte Genies, weil sich in deren Lebensläufen aufschlussreichere Informationen finden lassen als in denen von Geisteswissenschaftlern, meinte Elbogen. ${ }^{2}$ Sicher hat das damit zu tun, dass letztere weniger bereit sind, über ihre Kindheit und Jugend Auskunft zu geben. Immanuel Kant z.B. hat sich dies ,aufs inständigste und ernstlichste " verbeten. ${ }^{3}$ Wie die meisten Geisteswissenschaftler wollte auch Kant sein Werk ins Zentrum der öffentlichen Aufmerksamkeit rücken und hielt seinen persönlichen Werdegang für zu unwichtig oder auch gar peinlich. Dies leistet natürlich der Bezeichnung „Genie“ ungemeinen Vorschub; denn je weniger über den Menschen privat bekannt ist, desto eher kann man den Autor bedeutender Werke als genial bezeichnen. Elbogens Versuch, den Begriff des Genies zu entmythologisieren, hat zunächst mein Interesse an diesem Thema geweckt, jedoch meine Erwartungen nur zum Teil erfüllt. Das mag daran liegen, dass er sich auf Ausschnitte bereits vorliegender biografischer Texte beschränkte und kaum eigene Reflexionen über das Thema formulierte. Deshalb bleibt das Genialische der porträtierten Figuren nur unzureichend hinterfragt und deshalb als Mythos weiterbestehen. Dennoch ermöglichten mir Elbogens

1 Elbogen, Paul: Genius im Werden, Die Jugend großer Menschen, Hamburg 1963

2 Ebd.: S. 6

3 Ebd.: S. 97 
Porträts, aus Erläuterungsgründen, auf diese hin und wieder zurückzugreifen.

Um meiner Neugierde bezüglich der Menschen, die sich hinter dem Mythos Genie verbergen, weiter zu folgen, habe ich mir Informationsmaterial beschafft, das mir mehr Aufschluss über den Werdegang von Intellektuellen geben könnte. Ich ging auf die Suche nach Biografien, Interviews, Statements und Ähnlichem, um auf den Grund der Entwicklung zum Intellektuellen oder zum sogenannten Genie zu gelangen.

Ausgangspunkt und Ansporn war der Epilog von Thomas Jung „Der Name des Intellektuellen“ in dem Buch „Fliegende Fische, eine Soziologie des Intellektuellen in 20 Porträts.“4 Hier resümiert er, dass es keine Möglichkeit gibt, den Begriff des Intellektuellen allgemein zu bestimmen. Erst indem der Intellektuelle einen identifizierbaren $\mathrm{Na}$ men trägt, wie z.B. Adorno, kann man sagen, dass es sich um einen Intellektuellen handelt. „Indem der Intellektuelle einen Namen bekommt, indem er in ein identifizierendes System der Bestellung eingestellt wird, kann der Begriff des Intellektuellen - als Zeichen wie als Personenname gleichermaßen - zum Allgemeinen werden. Der Intellektuelle wird zum Namen, weil der Name das Bezeichnete erst kenntlich macht, was das bloße Zeichen ,Intellektueller' noch offenlässt. Weil der Name ,Intellektueller' auch Eigenname ist, wird dessen phantasmatisches Versprechen auf Singularität, auf Einzigartigkeit zugleich zum Phantasmatischen eines Namens des Intellektuellen: Besonderheit im Allgemeinen bzw. öffentliche Auserwähltheit."5 Damit behauptet Jung, dass sich der Begriff des Intellektuellen a priori nirgendwo herleiten lässt, sondern nur a posteriori durch die individuelle Verkörperung einer bestimmten Person erkenntlich wird. Dass diese bestimmte Person eine Vita besitzt, die allen Intellektuellen ähnlich ist, die Mühe, dies zu untersuchen, machte er sich nicht. Dabei steht er nicht allein. Wie üblich begnügte er sich mit der Variante der "Auserwähltheit“, was den Intellektuellen wieder in die Sphäre des Genies rückt, dem seine besondere Begabung in die Wiege gelegt ist und keiner weiteren Erklärung bedarf. Als ob er kein normaler Mensch wäre, den lediglich

4 Jung, Thomas/Stefan Müller-Doohm(H.g.): Fliegende Fische, Eine Soziologie des Intellektuellen in 20 Porträts, Frankfurt a.M. 2008

5 Ebd.: S. 454 
seine besondere Sozialisation, die durchaus nicht beneidenswert sein muss, zum Intellektuellen, zum Genie werden lässt. Jung zäumt das Pferd von hinten auf, wenn er erklärt, dass man einen Intellektuellen erst definieren kann, wenn er einen bestimmten Namen trägt. In Wirklichkeit ist es umgekehrt: es trägt dieser Mensch erst seinen bekannten Namen, wenn er seine besondere Sozialisation durchlaufen hat. Jung hilft sich aus der Widersprüchlichkeit seiner These, indem er einen dialektischen Trick anwendet: „Der Name des Intellektuellen beinhaltet eine doppelte Inskription: allgemeiner und zugleich besonderer, einzigartiger Name in Gestalt des anerkannten Eigennamens zu sein - auch Renommee genannt. "6 Indem der Name Adorno mit dem Begriff des Intellektuellen übereinstimmt, sind beide Identisch. Damit spricht er all den vielen namentlich unbekannten Intellektuellen, deren Namen erst viel später oder vielleicht nie das Licht der Öffentlichkeit erblicken, ihre gegenwärtige Besonderheit ab. Für Jung zählt nur der Bekanntheitsgrad des Namens, um einen Intellektuellen zu identifizieren. Die Gründe für seine Herkunft aus, im besonderen Maße, dem gehobenen Kleinbürgertum, bleiben im Dunkeln. „Der Name, zumal der Eigenname, will retten, was im Designationssystem notwendigerweise verschwindet: die Singularität."7 Jung geht so weit, dass er behauptet, dass der Begriff gar nicht abgeleitet werden kann: „Der Name des Intellektuellen präsentiert, kehrt hervor, was das Zeichen ,Intellektueller' verweigert: Benennung dessen, was nicht benannt sein kann und will...Man kann auch sagen: Der Name des Intellektuellen leistet eine Identifizierung von eigentlich Nichtidentifizierbarem, bzw. anders formuliert: Der Begriff ,Intellektueller` erhält über den Namen, was er als zeichenhafte Leerstelle nicht einzuholen vermag: eindeutige Bedeutungszuweisung, d.h. designative Erfüllung. " ${ }^{8}$ Jung spricht sogar von einem „Identifizierungsverbot“ des Begriffs des Intellektuellen, womit er sein Unbehagen verdrängen möchte, weil er über den Begriff des Intellektuellen nichts herausgefunden hat. Deshalb verwundert es auch nicht, dass er, in Übereinstimmung mit seinem Mitherausgeber Stefan Müller-Doohm, auf Hegel zurückgreift und mit diesem seine Argu-

6 Ebd.: S. 454

7 Ebd.

8 Ebd.: S. 453

9 Ebd.: S. 453 
mentation untermauert: Hegel insistiert nämlich darauf, „dass man Intellektuelle nicht nach ihren Tugenden, nach ihrem praktischen Engagement beurteilen könne, noch weniger nach ihren weltgeschichtlichen Diagnosefähigkeiten, sondern allein dadurch, dass man die ihrem Denken zugrundeliegenden Deutungsmuster erfasst."10 Unter diesem Deutungsmuster verstehen Jung und Doohm den persönlichen Namen desjenigen, der in der Öffentlichkeit als Intellektueller gilt. Wie Hegel, der einen ,absoluten Geist', der an sich unbestimmbar ist und sich partiell nur in der Vernunft eines einzelnen, gebildeten Menschen und seinen Taten erkennbar macht, in der Welt voraussetzt, verstehen beide Autoren den Intellektuellen. Er kann sich nur in bestimmten, besonders gebildeten Menschen manifestieren, als ob sich Intellektualität freischwebend wie der absolute Geist Hegels in der Welt herumtreibt und eine geeignete Person für seine intellektuelle Mission sucht, um sich zu manifestieren. Als ob sich der Mensch nicht selbst und unter größten reflexiven Anstrengungen zu einem Intellektuellen entwickeln könnte, „dies alles im Bewusstsein, dass jeder Versuch einer approximativen Bestimmung des Sozialtyps ,Intellektueller' eben nur eine versuchsweise Annäherung und keine fertiggestellte Attribuierung sein kann." Das ist reinster Hegelscher Idealismus, der dem menschlichen Subjekt als solchem auch keine intellektuelle Erkenntnisleistung zutraute und deshalb einen absoluten Geist erfand, der sich reflexiv herausragender Individuen bedient, um den menschlichen Fortschritt zu betreiben. Deshalb suchen die Autoren in den Werken von z.B. Max Weber, Karl Mannheim, Adorno, Habermas, Jaspers u. a. nach Spuren des ,Weltgeistes', sprich Intellektualität, können ihn aber nicht finden.

Deshalb bezeichnet auch der Titel ihres Buches „Fliegende Fische“, der zunächst absurd anmutet, das ganze Ergebnis ihrer Recherchen, nämlich einen hilflosen Rekurs auf Voltaires Metapher der fliegenden Fische, mit der dieser die Unbestimmbarkeit, die Leerstelle des Begriffs ,Intellektueller' typisierte.

Um Jungs Begriff der „Leerstelle“, mit dem er die Unbestimmbarkeit des Intellektuellen -Typus bedeuten will, zu füllen, sollen die folgenden Ausführungen dienen. 
